\title{
A Novel GNSS Repeater Architecture for Indoor Positioning Systems in ISM Band
}

\author{
Abdulkadir Uzun, Firas Abdul Ghani, Hüsnü Yenigün, İbrahim Tekin \\ Electronics Engineering, Sabanci University \\ Istanbul, Turkey \\ \{kadiruzun, firas, yenigun, tekin\}@ sabanciuniv.edu
}

\begin{abstract}
For indoor positioning using customary Global Navigation Satellite Systems (GNSS) receivers, the use of GNSS repeaters can be considered. However, regulations and provisions limit the use of GNSS repeaters. In order to circumvent these limitations, we propose a novel repeater architecture that transmits the Global Positioning System (GPS) signals in $433 \mathrm{MHz}$ ISM band by frequency down-conversion. In addition, a receiver front end is proposed to upconvert GPS signals back to $1575.42 \mathrm{MHz}$, which allows any off-the-shelf GPS receiver to be used for indoor positioning. Measurement results with the RF hardware show that when the GPS signals are downconverted and then upconverted back to its original frequency, GPS signal fidelity and positioning accuracy are preserved.
\end{abstract}

Keywords-GPS repeater; indoor positioning; ISM band

\section{INTRODUCTION}

Global indoor positioning market is forecasted to expand by $42 \%$ compound annual growth rate from 2017 to 2023 [1]. The forecasted growth promises the requirement for newer technologies and methods in the field. One of the existing methods for indoor positioning is through the repeaters.

GPS operates with an accuracy of 3 meters or better in horizontal and 5 meters in vertical dimension [2]. Through GNSS repeaters GPS signals are amplified and utilized to track the location of anything carrying a customary receiver indoors. However, the use of GNSS repeaters is restricted to prevent repeaters from interfering with other uses of GNSS in the vicinity. The Electronic Communications Committee's (ECC) Reports 129 [3] and 145 [4], and European Telecommunication Standards Institute's (ETSI) standard EN 302645 [5] prohibit the overall amplification of GPS signals more than $45 \mathrm{~dB}$, and the antenna gain exceeding $3 \mathrm{~dB}$ as it otherwise would affect nearby systems using GNSS services such as aeronautical radio navigation system DME, military and civilian radars, Earth Exploration Satellite Service, and so on. The US policy [2] presents that GPS repeaters can be used only by the departments of the US Federal Government or those who will deploy the system within the shielded indoor environment or have a license under FCC.

Existing systems $[6,7]$ deploy at least three repeaters that do not interfere with each other and amplify the GPS signal picked by directional antennas for $2 \mathrm{D}$ positioning. In this paper, we present a novel GPS repeater system operating in $433 \mathrm{MHz}$ ISM band, which is free from the legal restrictions, to replace the repeater architecture in [7]. The transceiver side down-converts the GPS signal from $1575.42 \mathrm{MHz}$ to $433 \mathrm{MHz}$ and amplifies the signal in the ISM band, while the receiver side amplifies the signal at $433 \mathrm{MHz}$ and then upconverts it to $1575.42 \mathrm{MHz}$ to be processed by the customary GPS receiver. By deploying at least three transceivers that replace the repeaters and an additional receiver for each receiver, one can achieve 2D positioning indoors. Another advantage of the proposed system is that the signal at $433 \mathrm{MHz}$ undergoes less free space path loss (FSPL) [8]. As the frequency changes from $1575.42 \mathrm{MHz}$ to $433 \mathrm{MHz}$, the FSPL decreases by $11.22 \mathrm{~dB}$.

In order to analyze the system performance, the transceiver and receiver sides are brought together and connected by an RF cable. The GPS signal is supplied by a signal generator to the input of the transceiver. The signal at the output of the receiver is processed in MATLAB with the algorithms proposed in [9]. GPS algorithms used in this study require further modifications as non-line-of-sight (NLOS) propagation through the repeater system is not considered. The result is compared with the location detected without the proposed hardware. The results of the experiment reveal out that once the GPS signal is downconverted to $433 \mathrm{MHz}$, the power level of the transmitted signal may be adjusted avoiding the regulations for the GNSS repeaters.

\section{HARDWARE}

The hardware of the proposed architecture has a transceiver and a receiver side. Picking up the GPS signals, the transceiver side down-converts the GPS signals and amplifies the signal indoors. The receiver side picks up the signal at 433 $\mathrm{MHz}$ and upconverts the signal back to $1575.42 \mathrm{MHz}$ to be processed by a customary GPS receiver.

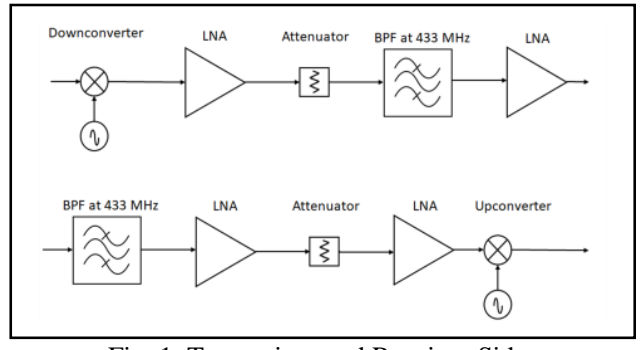

Fig. 1. Transceiver and Receiver Sides

The proposed transceiver design is demonstrated in Fig.1. It consists of a bias tee to bias the outdoor GPS active antenna, a downconverter board ADRF6820-EVALZ with a conversion 
loss of $8.7 \mathrm{~dB}$, a low noise amplifier (LNA) LHA13-LN+ with $22.4 \mathrm{~dB}$ gain, $20 \mathrm{~dB}$ attenuator by cascading two VAT-10+ components, another LNA LHA-13LN+ and finally a $2 \mathrm{MHz}$ bandpass filter with $1.7 \mathrm{~dB}$ insertion loss at $433 \mathrm{MHz}$. The overall expected gain of the downconverter is $14.8 \mathrm{~dB}$.

The proposed receiver design is also given in Fig.1. The receiver consists of a bandpass filter with $1.7 \mathrm{~dB}$ insertion loss and $2 \mathrm{MHz}$ bandwidth at $433 \mathrm{MHz}$, the LNA LHA13-LN+ with the gain of $22.4 \mathrm{~dB}, 20 \mathrm{~dB}$ attenuator by cascading two VAT-10+ components, another LHA-13LN+, bias tees required for upconverter $\mathrm{RF}$ inputs and the upconverter board ADRF6720-27-EVALZ with a total power loss of $1.3 \mathrm{~dB}$. The overall expected gain is $21.8 \mathrm{~dB}$.

\section{RESULTS}

Previously, the transceiver and the receiver sides are explained with the novelty of the approach being the propagation of GPS signals in the $433 \mathrm{MHz}$ ISM band. For this study, GPS signal at $1575.42 \mathrm{MHz}$ with $-81.6 \mathrm{dBm}$ power is supplied to the input of the transceiver from the Rohde \& Schwarz SMBV100A vector signal generator. The transceiver and receiver are connected by an RF cable. The output signal power is measured with Rohde \& Schwarz FSH8 Spectrum Analyzer for $300 \mathrm{kHz}$ resolution and $20 \mathrm{MHz}$ channel bandwidth. A customary GPS receiver is deployed after the receiver side. The measurement setup and the path that signal flows is shown in Fig 2. The results in Table I overlap with the expected gain values.

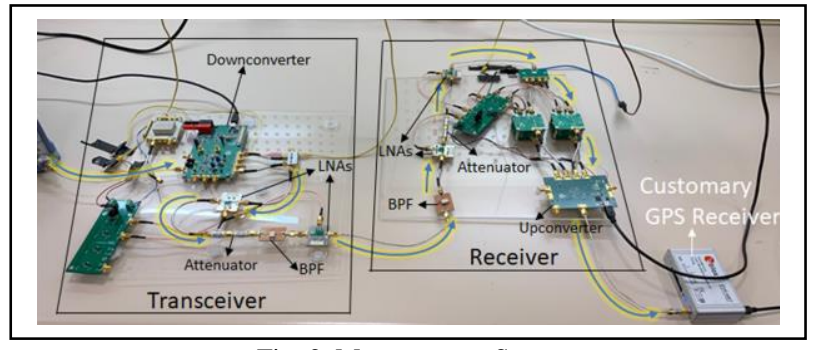

Fig. 2. Measurement Setup

TABLE I. POWER LeVElS AT THE OUTPUT OF SyStem Blocks

\begin{tabular}{|l|c|c|c|}
\hline \multirow{2}{*}{$\begin{array}{l}\text { Measurement } \\
\text { Points }\end{array}$} & \multicolumn{3}{|c|}{ Power Levels } \\
\cline { 2 - 4 } & $\begin{array}{c}\text { Power over 20 } \\
\text { channel (dBm) }\end{array}$ & $\begin{array}{c}\text { Peak } \\
(\mathbf{d B m} / \mathbf{H z})\end{array}$ & Gain $(\boldsymbol{d B})$ \\
\hline Transceiver Input & -81.6 & -144.3 & - \\
\hline Transceiver Output & -66.7 & -131.8 & 14.9 \\
\hline Receiver Output & -45.9 & -111.3 & 20.8 \\
\hline
\end{tabular}

When the GPS signal level is $-100 \mathrm{dBm}$ at the input of the system shown in Fig. 2, the detected location using the algorithms in [9] is depicted in Fig. 3 with the green stars. The center of the circle is the reference location provided by the signal generator. The red stars in Fig. 3 represent the location found when the GPS signal generator is connected directly to an off-the-shelf GPS receiver. Averaging over 100 samples, the error obtained with and without hardware are $106 \mathrm{~cm}$ and $119 \mathrm{~cm}$, respectively. The obtained results are summarized in Table II. The circular error probable is 8.53 meters from the estimated average position with hardware.

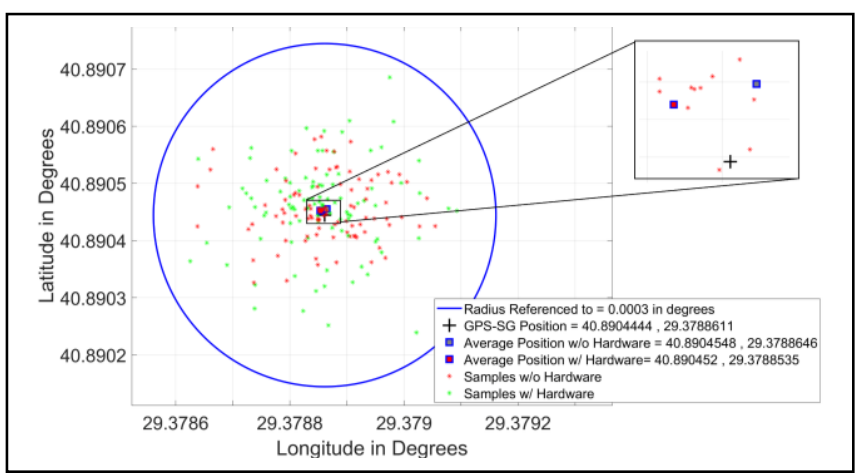

Fig. 3. Latitude and Longitude found w/ and w/o the proposed hardware

TABLE II. COMPARISON OF THE DETECTED AND REFERENCE POINTS

\begin{tabular}{|l|c|c|c|}
\hline & Latitude & Longitude & $\begin{array}{c}\text { Distance from the } \\
\text { reference }\end{array}$ \\
\hline Reference & 40.8904444 & 29.3788611 & $0 \mathrm{~cm}$ \\
\hline $\begin{array}{l}\text { without Hardware } \\
\text { (average) }\end{array}$ & 40.8904548 & 29.3788646 & $119 \mathrm{~cm}$ \\
\hline $\begin{array}{l}\text { with Hardware } \\
\text { (average) }\end{array}$ & 40.8904520 & 29.3788535 & $106 \mathrm{~cm}$ \\
\hline
\end{tabular}

\section{CONCLUSION}

The proposed repeater system can be used for indoor applications as GPS signal integrity is preserved after passing through the system. It allows the use of customary GPS receivers and existing algorithms with a modification to include NLOS effects. This system is not bounded by the regulatory framework for the use of GNSS repeaters as the system works at $433 \mathrm{MHz}$ in the ISM band. Lower path loss that the signal is exposed is another advantage of the system.

\section{ACKNOWLEDGMENT}

This study is supported by the Scientific and Technological Research Council of Turkey (TÜBITTAK), Grant No: 116E752.

\section{REFERENCES}

[1] KBV research (2017). Global Indoor Location Market

[2] U.S. Department Of Commerce National Telecommunications and Information Administration, "Manual Of Regulations And Procedures For Federal Radio Frequency Management," September 2017.

[3] ECC, "Technical and Operational Provisions Required for the Use of GNSS Repeaters," Dublin, 2009.

[4] ECC, "Regulatory Framework for Global Navigation Satellite System (GNSS) Repeaters," St. Petersburg, 2010.

[5] ETSI, "Electromagnetic compatibility and Radio spectrum Matters (ERM); Short Range Devices; Global Navigation Satellite Systems (GNSS) Repeaters; Harmonized EN covering the essential requirements of article 3.2 of the R\&TTE Directive," 2010.

[6] I. Tekin, A. Bozkurt, K. Ozsoy, "Indoor Positioning System Based on GPS Signals and Pseudolites with Outdoor Directional Antennas," U.S. Patent US20120286992A1

[7] K. Ozsoy, A. Bozkurt, I. Tekin, "Indoor Positioning Based on Global Positioning System Signals", Microwave and Optical Technology Letters 55.5 (2013): 1091-1097.

[8] H.T. Friis, "A Note on a Simple Transmission Formula," IRE Proc.: 254-256, May 1946.

[9] K. Ozsoy, A. Bozkurt, I. Tekin, '2D Indoor positioning system using GPS signals", IPIN 2010, Zürich, Switzerland, September 2010. 